\title{
WINDOW SCALING IN ONE-DIMENSIONAL MAPS
}

\author{
T. POST, H.W. CAPEL \\ Instituut voor Theoretische Fysica, Universiteit van Amsterdam, Valckenierstraat 65, 1018 XE Amsterdam, The Netherlands
}

and

J.P. VAN DER WEELE

Centrum voor Theoretische Natuurkunde, Universiteit Twente, Postbus 217, 7500 AE Enschede, The Netherlands

Received 17 November 1988; accepted for publication 15 December 1988

Communicated by D.D. Holm

\begin{abstract}
We describe both the internal structure and the width of the periodic windows in one-dimensional maps, by considering a universal local submap. Both features are found to depend only on the order of the extremum of this submap. Moreover, we discuss how the windows are grouped in accumulating families, and we calculate the scaling of the widths within these families.
\end{abstract}

\section{Introduction}

Windows in chaos are often encountered, both in theory and experiments [1-3]. It has been claimed $[4,5]$ that the internal structure of windows is always such that the ratio $\gamma$ between the total window width and the distance from the opening of the window to the first period-doubling bifurcation is equal to $9 / 4$. However, in experiments values of $\gamma$ are observed which significantly differ from $9 / 4$ [6-8]. This discrepancy can be explained by noting that Yorke et al. have restricted themselves to mappings with a quadratic $(z=2)$ maximum. The same restriction is present in previous investigations on the scaling of window widths $[9,10]$. In a general situation we must allow also for other values of $z$, as exemplified by the map

$f(x)=1-a|x|^{z}, \quad z>1$.

In fig. 1 we have shown a part of the bifurcation tree of this map for $z=5$. The ratio $\gamma$ is here equal to 1.29.

In section 2 we argue that every window can be described by a universal local submap of order $z$, and we use this to calculate the ratio $\gamma$ as a function of $z$.

In section 3 we obtain an expression for the width of an arbitrary window of period $n$.

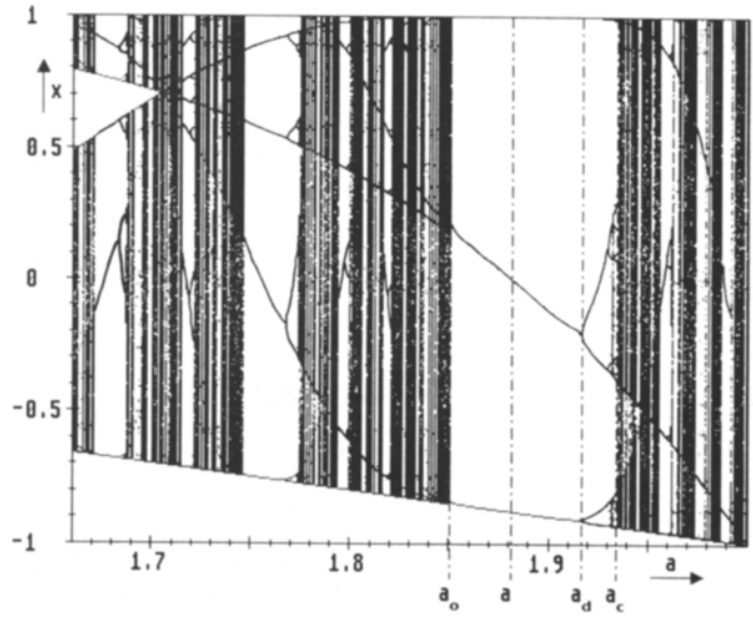

Fig. 1. A part of the bifurcation tree for the map $f(x)=1-a|x|^{2}$, in the case $z=5$. For the period-3 window we have indicated the values $a_{\mathrm{o}}$ and $a_{\mathrm{c}}$ associated with the opening and the closing of the window, as well as the value $a_{\mathrm{d}}$ where the first period doubling occurs. The ratio $\gamma$ discussed in the text is given by $\left(a_{\mathrm{c}}-a_{\mathrm{o}}\right) /$ $\left(a_{\mathrm{d}}-a_{\mathrm{o}}\right)$. Also indicated is the value $\tilde{a}$ where the 3 -cycle is superstable.

Subsequently, in section 4 , we consider families of windows which accumulate with increasing period $n$ at certain values of the parameter $a$. For these fam- 
ilies we derive an exact scaling relation, which tells us how the widths decrease with growing $n$.

\section{The normalized submap}

In order to understand the features of a period- $n$ window we consider the $n$ times iterated mapping $f^{n}(x)$. For example, for the 3-window we consider $f^{3}(x)$, as plotted in fig. 2 , for $z=3$.

Every extremum of $f^{n}(x)$ is again of order $z$, so for instance the central extremum can be locally described by

$x_{n(i+1)}=\lambda\left|x_{n i}\right|^{2}+\mu$.

Substituting

$x_{n i}-\tilde{x}_{0}=u_{i}|\lambda|^{-1 /(z-1)} \operatorname{sgn} \lambda$,

$\mu=-m|\lambda|^{-1 /(z-1)} \operatorname{sgn} \lambda$,

we obtain the normalized submap

$u_{i+1}=\left|u_{i}\right|^{2}-m$.

This submap describes fully the behaviour of an orbit within the period- $n$ window. Note that $m$ is linearly related with the parameter $a$ of the original map (1), which means that any ratio in terms of $a$ corresponds to the same ratio in terms of $m$, assuming

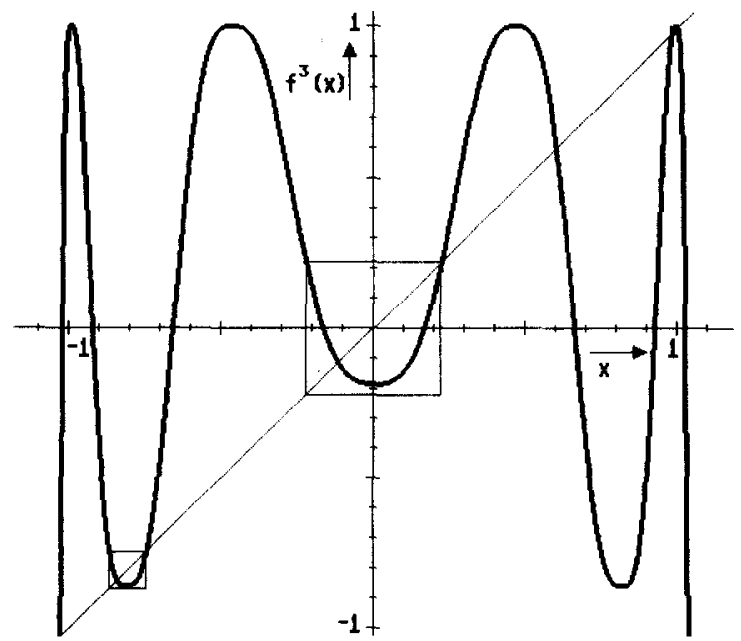

Fig. 2. The $\operatorname{map} f^{3}(x)$ for $z=3$ at $a=1.86$. The boxes indicate the regions of the submaps discussed in the text. The smallest box, near $x=1$, is not discernible in this figure. of course that the window is small enough to make a local description applicable. We shall give the explicit form of this linear relation in the next section.

In fig. 3 we have drawn the normalized submap for $z=4$ at several values of $m$, which will be used in this paper.

The window is opened at $m=m_{0}$, where the submap is tangent to the line $u_{i+1}=u_{i}$. Its value is easily calculated to be

$m_{\mathrm{o}}=z^{-1 /(z-1)}\left(z^{-1}-1\right)$.

Superstability is attained at $\tilde{m}=0$. The next special value of $m$ corresponds to the first period-doubling bifurcation:

$m_{\mathrm{d}}=z^{-1 /(z-1)}\left(z^{-1}+1\right)$,

when the submap intersects the line $u_{i+1}=u_{i}$ with slope -1 . Finally the closing of the window takes place when the orbit can escape from the region (box) of the local submap. This occurs at

$m_{\mathrm{c}}=2^{1 /(z-1)}$.

From the above results we immediately obtain for the ratio $\gamma$ :

$\gamma=\frac{m_{\mathrm{c}}-m_{\mathrm{o}}}{m_{\mathrm{d}}-m_{\mathrm{o}}}=\frac{1}{2}\left[(2 z)^{1 /(z-1)}+1-z^{-1}\right]$.

For $z=2$ this yields indeed $\gamma=9 / 4$, but for other val-

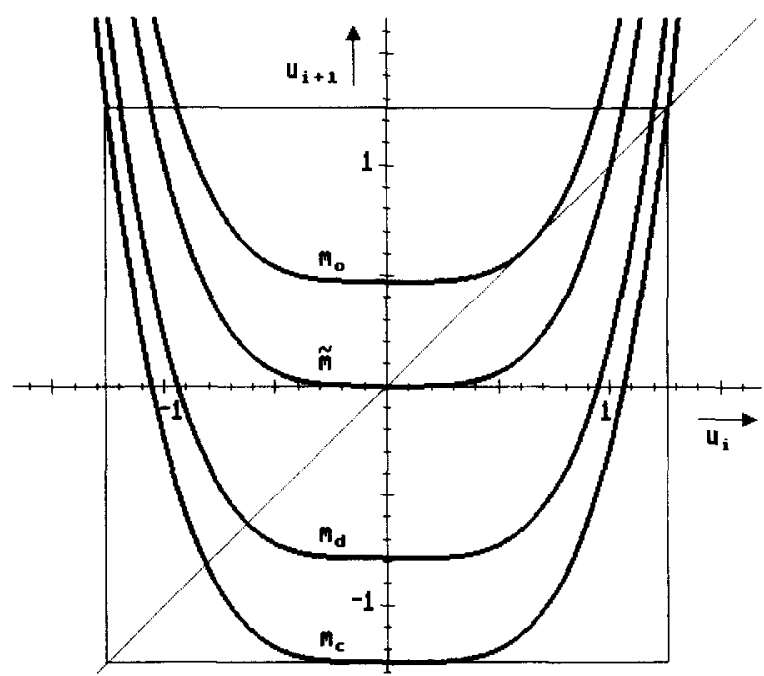

Fig. 3. The normalized submap $u_{n+1}=\left|u_{n}\right|=-m$ for $z=4$ at the values $m=m_{\mathrm{o}}, m=\tilde{m}, m=m_{\mathrm{d}}$ and $m=m_{\mathrm{c}}$. 
ues of $z$ the value of $\gamma$ varies from 1 to $+\infty$. For instance for $z=1.2$, reported from an experiment on chemical turbulence [11], we get $\gamma=39.9$, and for $z=1.1$ we have $\gamma=1328$. In fig. 4 we have plotted $\gamma$ as a function of $z$.

To test the validity of (9) for finite windows, we have presented the results of a direct numerical calculation of $\left(a_{\mathrm{c}}-a_{\mathrm{o}}\right) /\left(a_{\mathrm{d}}-a_{\mathrm{o}}\right)$ for the mapping (1) for $z=3$ in table 1 . These results should be compared to the analytical estimate $\gamma=1.558078 \ldots$, which follows from (9). It turns out that already for the $n=5$ windows the agreement is within 0.5 percent.

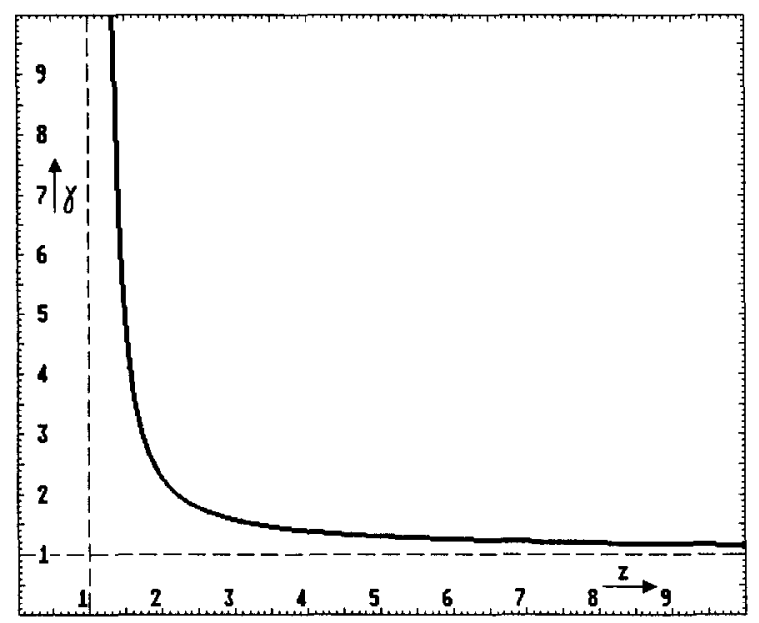

Fig. 4. The ratio $\gamma$ as a function of $z$.

\section{The width of a period- $n$ window}

In order to obtain a simple estimate of the width of a period- $n$ window we go back from the normalized submap to the local description of the map $f^{n}(x)$ given by (2). All we need is to find the coefficients $\lambda$ and $\mu$ in terms of $a$. This then yields the width $a_{c}-a_{\mathrm{o}}$ of a period- $n$ window:

$$
\begin{aligned}
a_{\mathrm{c}} & -a_{\mathrm{o}}=\frac{\mu_{\mathrm{c}}-\mu_{\mathrm{o}}}{\mathrm{d} \mu /\left.\mathrm{d} a\right|_{a-a_{n}}} \\
= & \frac{m_{\mathrm{c}}-m_{\mathrm{o}}}{-|\lambda|^{1 /(z-1)} \operatorname{sgn} \lambda \mathrm{d} \mu /\left.\mathrm{d} a\right|_{a=a_{n}}},
\end{aligned}
$$

$\tilde{a}_{n}$ being the value of $a$ where we have a superstable $n$-cycle, with cycle elements $\tilde{x}_{0}, \tilde{x}_{1}$, etc. In the second step we have used the substitution (4).

To determine $\lambda$ and $\mathrm{d} \mu / \mathrm{d} a$ we consider $f^{n}(x)$ in the neighbourhood of $a=\tilde{a}_{n}$. Expanding $f^{n}(x)$ around $\tilde{x}_{0}$ (which equals 0 for the map (1)), we get

$\lambda=-a L=-a \prod_{i=1}^{n-1} f^{\prime}\left(\tilde{x}_{i}\right)$.

Here the quantity $L^{1 /(n-1)}$ corresponds to the socalled reduced Lyapunov exponent introduced in ref. [4].

To find an expression for $\mathrm{d} \mu / \mathrm{d} a$ we observe that $\mu$ is the vertical distance of the central extremum of the map $f^{n}(x)$ to the line $x=y$, which must locally be equated to the small quantity $f^{n}\left(\tilde{x}_{0}\right)-\tilde{x}_{0}$.

Expanding around $a=a_{n}$ we have

Table 1

Numerical values of $\left(a_{\mathrm{c}}-a_{\mathrm{o}}\right) /\left(a_{\mathrm{d}}-a_{\mathrm{o}}\right)$ and of the width $\left(a_{\mathrm{c}}-a_{\mathrm{o}}\right)$ in comparison with the theoretical estimate according to (10), (11), (13), for several windows of the map $f(x)=1-a|x|^{3}$, labeled by the period $n$ and the value of $a$ where superstability is attained. The values of $\left(a_{\mathrm{c}}-a_{\mathrm{o}}\right) /\left(a_{\mathrm{d}}-a_{\mathrm{o}}\right)$ should be compared to $\gamma(3)=1.558078 \ldots$, obtained from (9).

\begin{tabular}{lllll}
\hline$n$ & $a_{n}$ & $\left(a_{\mathrm{c}}-a_{\mathrm{o}}\right) /\left(a_{\mathrm{d}}-a_{\mathrm{o}}\right)$ & $a_{\mathrm{c}}-a_{\mathrm{o}}$ & Estimated width \\
\hline 9 & 1.6244412677 & 1.56221 & $6.92576 \times 10^{-4}$ & $6.87617 \times 10^{-4}$ \\
7 & 1.6393215347 & 1.56191 & $3.98982 \times 10^{-3}$ & $3.95715 \times 10^{-3}$ \\
5 & 1.6859164319 & 1.55189 & $1.97357 \times 10^{-2}$ & $1.98226 \times 10^{-2}$ \\
3 & 1.8191725134 & 1.52052 & $6.69091 \times 10^{-2}$ & $7.08386 \times 10^{-2}$ \\
5 & 1.9149538511 & 1.55031 & $4.37745 \times 10^{-3}$ & $4.43110 \times 10^{-3}$ \\
4 & 1.9699611643 & 1.54469 & $4.53773 \times 10^{-3}$ & $4.64018 \times 10^{-3}$ \\
5 & 1.9950086217 & 1.55269 & $3.09031 \times 10^{-4}$ & $3.11878 \times 10^{-4}$ \\
6 & 1.9991688636 & 1.55586 & $2.10759 \times 10^{-5}$ & $2.11558 \times 10^{-5}$ \\
7 & 1.9998615079 & 1.55717 & $1.43626 \times 10^{-6}$ & $1.43849 \times 10^{-6}$ \\
8 & 1.9999769191 & 1.55770 & $9.78007 \times 10^{-8}$ & $9.78631 \times 10^{-8}$ \\
9 & 1.9999961532 & 1.55793 & $6.65681 \times 10^{-9}$ & $6.65855 \times 10^{-9}$ \\
\hline
\end{tabular}


$\mu=\left.\frac{\mathrm{d}\left[\tilde{x}_{n}(a)-\tilde{x}_{0}\right]}{\mathrm{d} a}\right|_{a=\tilde{a}_{n}}\left(a-\tilde{a}_{n}\right)+\mathrm{O}\left(\left(a-\tilde{a}_{n}\right)^{2}\right)$,

and after some algebra (see ref. [12]) we obtain

$$
\begin{aligned}
& \left.\frac{\mathrm{d} \mu}{\mathrm{d} a}\right|_{a=\tilde{a}_{n}}=\left.\frac{\mathrm{d} f^{n}\left(\tilde{x}_{0}-\tilde{x}_{0}\right)}{\mathrm{d} a}\right|_{a=\tilde{a}_{n}} \\
& \quad=\frac{1}{\tilde{a}_{n}}\left(-1+\sum_{j=2}^{n-1}\left(\tilde{x}_{j}-1\right) \prod_{i=j}^{n-1} f^{\prime}\left(\tilde{x}_{i}\right)\right),
\end{aligned}
$$

which can be used in combination with (10) and (11) to estimate the window width.

In table 1 we compare the estimate widths, using (11) and (13), of some important windows for $z=3$ with the results of a direct numerical calculation of $a_{\mathrm{c}}-a_{\mathrm{o}}$. It turns out that already for the $n=6$ windows there is agreement within 1 percent.

\section{Scaling within families of windows}

At several points $a=a^{*}$ in the bifurcation tree we observe that windows accumulate, with increasing period. Since a period- $n$ window is organized around the superstable period- $n$ cycle that lies within its borders, we can just as well speak of an accumulation of superstable cycles. The simplest accumulation point of this kind lies at $a=2$, where the $R L^{n-2}$ family of superstable cycles accumulates $[9,13]$. Each member of this family has one element $\left(\tilde{x}_{1}=1\right)$ at the right-hand side of $\tilde{x}_{0}$ and the next $n-2$ elements all lie at the left-hand side of $\tilde{x}_{0}$. The occurrence of this family can be understood by looking at fig. 5 , where we have depicted two successive members. In this figure we see that the extra periodic points are systematically added near the unstable fixed point $x^{*}$, in going from the superstable orbit in the $n$-window to the superstable orbit in the $(n+1)$-window.

Consequently we have from (11)

$\lambda(n)=A_{n}\left[f^{\prime}\left(x^{*}\right)\right]^{n}$,

where $A_{n} \rightarrow A$ in the limit of large $n$. Similarly, from eq. (13) it can be shown that

$\frac{\mathrm{d} \mu(n)}{\mathrm{d} a}=B_{n}\left[f^{\prime}\left(x^{*}\right)\right]^{n}$,

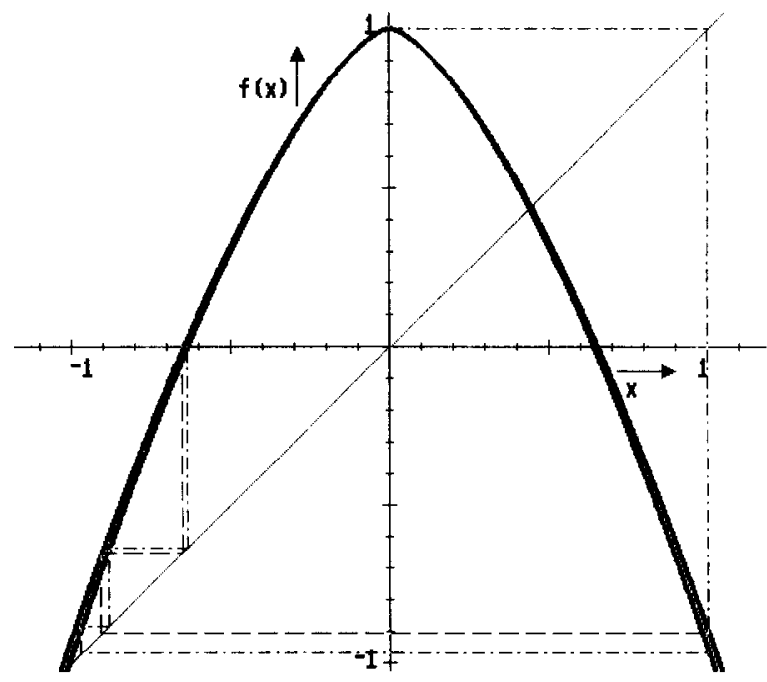

Fig. 5. The map $x_{n+1}=1-a\left|x_{n}\right|^{z}$ for $z=1.5$. The iterates of a superstable 4-cycle (dashed lines) and a superstable 5-cycle (dashdotted lines) are indicated. Extra iterates are added near the fixed point $x^{*}$

where $B_{n} \rightarrow B$ for large $n$. A more detailed derivation of eqs. (14) and (15), including an explicit evaluation of $A_{n}$ and $B_{n}$ will be presented elsewhere [12]. Eqs. (14) and (15), inserted in eq. (10), yield the following scaling relation for the width of the windows,

$\lim _{n \rightarrow \infty} \frac{\text { width }(n-1)}{\text { width }(n)}=\left|f^{\prime}\left(x^{*}\right)\right|^{z /(z-1)}$.

For our map (1) at $a=2$ we have $x^{*}=-1$ and hence $f^{\prime}\left(x^{*}\right)=2 z$. A similar scaling, which is however only valid for $z=2$, was reported in ref. [9].

Another family of accumulating windows is located near $a=a_{1}(z)$, where $a_{1}$ is the parameter value where the two-band chaotic attractor becomes a oneband attractor. The members of this family are $R L R^{n-3}[10,13]$. That is, the first element $\left(\tilde{x}_{1}=1\right)$ is at the right-hand side of $\tilde{x}_{0}$, the second one $\left(\tilde{x}_{2}=1-a\right)$ at the left, and all the other $n-3$ elements lie again at the right-hand side of $\tilde{x}_{0}$. The successive members of this family are situated alternatingly above and below $a=a_{1}$, i.e. the odd members accumulate at $a_{1}$ from above and the even members accumulate from below.

For this family the extra periodic points for increasing $n$ are systematically added near the positive 
Table 2

Numerically obtained width ratio factors for the window families $R L^{n-2}$ and $R L R^{n-3}$ of the map $f(x)=1-a|x|^{z}$, for various values of $z$, as compared to the exact (limiting) scaling factors given by (16) and (17).

\begin{tabular}{|c|c|c|c|c|c|c|c|}
\hline \multirow[t]{2}{*}{$n$} & \multicolumn{3}{|l|}{$R L^{n-2}$} & \multirow[t]{2}{*}{$n$} & \multicolumn{3}{|c|}{$R L R^{n-3}$} \\
\hline & $z=1.5$ & $z=2$ & $z=3$ & & $z=1.5$ & $z=2$ & $z=3$ \\
\hline 5 & 32.914 & 16.920 & 14.684 & 18 & 3.7018 & 2.7968 & 2.5246 \\
\hline 6 & 22.996 & 16.312 & 14.663 & 19 & 3.9060 & 2.8308 & 2.5242 \\
\hline 7 & 27.903 & 16.099 & 14.674 & 20 & 3.7671 & 2.8094 & 2.5253 \\
\hline 8 & 27.353 & 16.030 & 14.686 & 21 & 3.8609 & 2.8228 & 2.5240 \\
\hline 9 & 27.14 & 16.009 & 14.692 & 22 & 3.799 & 2.8144 & 2.5252 \\
\hline 10 & 27.1 & 16.00 & 14.695 & 23 & 3.84 & 2.820 & 2.5241 \\
\hline $\begin{array}{l}\infty \\
\text { (exact) }\end{array}$ & 27 & 16 & 14.697 & $\begin{array}{l}\infty \\
\text { (exact) }\end{array}$ & 3.8221 & 2.8176 & 2.5246 \\
\hline
\end{tabular}

fixed point $X$. The same argument as above yields the scaling relation

$\lim _{n \rightarrow \infty} \frac{\text { width }(n-1)}{\text { width }(n)}=\left|f^{\prime}(X)\right|^{z /(z-1)}$.

For our map $f(x)=1-a|x|^{z}$ at $a=a_{1}$ we have $X=a_{1}-1$ and hence $f^{\prime}(X)=z\left(2-a_{1}\right) /\left(a_{1}-1\right)$, see also ref. [14]. Similar scaling expressions can be derived for other accumulating window families.

In table 2 we present some numerically obtained width ratios for both families, for various values of $z$. The deviations from the exact (limiting) values given by (16) and (17) are due to the $n$-dependence of the amplitudes $A_{n}$ and $B_{n}$ in eqs. (14) and (15). An extended discussion of this $n$-dependence will be given in the future [12]. In ref. [10] a numerically obtained limiting width ratio $7.9384 \ldots$ was reported for successive odd members of the $R L R^{n-3}$ family, which accumulate at $a=a_{1}$ from above. This result indeed corresponds up to four decimals to our squared exact limiting value $\left|f^{\prime}(X)\right|^{2 z /(z-1)}=$ $7.938918 \ldots$ for $z=2$.

\section{References}

[1] P. Collet and J.-P. Eckmann, Progress in phyises, Vol. 1. Iterated maps on the interval as dynamical systems (Birkhäuser, Basel, 1980).

[2] P. Cvitanović, ed., Universality in chaos, a reprint selection (Hilger, Bristol, 1984).

[3] C. Grebogi, E. Ott and J.A. Yorke, Physica D 7 (1983) 181.

[4] J.A. Yorke, C. Grebogi, E. Ott and L. Tedeschini-Lalli, Phys. Rev. Lett. 54 (1985) 1095.

[5] J. Dias de Deus, R. Dilão and A. Noronha da Costa, Phys. Lett. A 124 (1987) 433.

[6] T. Matsumoto, L.O. Chua and S. Tanaka, Phys. Rev. A 30 (1984) 1155

[7] M.J. Hasler, Proc. IEEE 75 (1987) 1009.

[8] T. Matsumoto, Proc. IEEE 75 (1987) 1033.

[9] J. Dias de Deus, R. Dilão and A. Noronha da Costa, preprint CERN/SPS/88-11 (1988).

[10] R. Brown, C. Grebogi and E. Ott, Phys. Rev. A 34 (1986) 2248.

[11] Y. Kuramoto and S. Koga, Phys. Lett. A 92 (1982) 1.

[12] T. Post, H.W. Capel and J.P. van der Weele, in preparation.

[13] N. Metropolis, M.L. Stein and P.R. Stein, J. Comb. Th. 15 (1973) 25 (also reprinted in ref. [2]).

[14] T. Post, H.W. Capel and J.P. van der Weele, Phys. Lett. A $133(1988) 373$. 